\title{
Multiple paragangliomas involving carotid body and vagal region: A sporadic case
}

\author{
Jaimanti Bakshi*, Atul Kumar Goyal, Dharam Vir and Naresh K Panda \\ Department of Otolaryngology and Head Neck Surgery (ENT), Post Graduate Institute of Medical Education and Research (PGIMER) Chandigarh, India
}

\begin{abstract}
Paragangliomas are rare neuroendocrine tumors also known to occur in head and neck regions and generally involve the carotid body or vagal region. Till date, only a single study has reported the occurrence of paragangliomas involving both carotid body and vagal region. The present study, to our best knowledge, is the second study in the world which reported the multiple paragangliomas involving the carotid body and vagal region. The patient in the present case study was a 45 years old male with a single swelling in the left upper part of the neck. In the pre-operative clinical examination, the swelling was identified as carotid body tumor, and the patient was then planned for surgery. The intra-operative findings revealed that it involved both the vagal and carotid body and was subsequently operated successfully. Postoperatively the swelling was described as bilateral carotid paragangliomas with left vagal paraganglioma.
\end{abstract}

\section{Introduction}

Paragangliomas are neuroendocrine tumors that represent $0.012 \%$ of all tumors [1]. Paragangliomas arise from extra-adrenal paraganglia, microscopic islands of cells derived embryonically from the neural crest cells of the autonomic nervous system $[1,2]$. Paragangliomas may occur anywhere from the skull base to the pelvic floor along with the distribution of paraganglia [3]. The common sites of occurrence include abdomen, retroperitoneum, chest and mediastinum and various head and neck locations [4,5]. In head and neck region, paragangliomas are very rare and represent $0.6 \%$ of all tumors [1] and found to occur in jugulotympanic membrane, orbit, nasopharynx, larynx, vagal body and carotid body regions $[5,6]$.

Carotid body tumors are the most common paraganglioma often present as slow growing, non- tender neck masses located just anterior to the sternocleidomastoid muscle at the level of the hyoid [4]. Carotid body paragangliomas arise at the bifurcation of the internal and external carotid arteries [5]. Vagal paraganglioma tumors arise from nests of paraganglionic tissue within the perineurium at various sites of the vagal nerve $[7,8]$. As opposed to carotid body tumors, vagal paragangliomas are located more cephalad in the neck, between the jugular vein and the internal carotid artery, sometimes extending to the base of the skull through the jugular foramen or posteriorly to the mastoid tip [7].

All types of paragangliomas are generally recommended be removed earliest possible because their development will involve contiguous structures, with difficult excision andpostoperative cranial nerve damage [6]. Surgical excision of the tumor includes proximal and distal control of the carotid arteries with ligation of feeding branches from the internal carotid arteries and dissection of the mass starting at the carotid bifurcation and continuing in a caudal-cranial fashion [6]. Elective external carotid arteries ligation can be helpful for tumor mobilization [6]. Alternatively, the dissection of the tumor can be started cranially which can facilitate early proximal control over most adjacent nerves, reducing the risk of postoperative morbidity [6].
The present case report represents the sporadic case of multiple paragangliomas involving the carotid body and vagal region. Till date, an only a single study has reported the occurrence of paragangliomas involving both the regions [6]. The present study, to our best knowledge, is the second study in the world which reported the multiple paragangliomas involving the carotid body and vagal region.

\section{Methodology}

\section{Recruitment of the patient}

The patient in present case report was recruited in the otolaryngology and head and neck surgery outpatient department (ENT-OPD) of the postgraduate institute of medical education and research (PGIMER), Chandigarh. Written consent was obtained from the patient and patient was also addressed about the numerous potential complications associated with the paraganglioma surgery. The patient was informed about the possible neural dysfunction with associated hoarseness, vocal change, aspiration, dysphagia, dysarthria, facial asymmetry, and shoulder weakness in addition to the possibility of a cerebrovascular accident.

\section{Case report}

\section{Sociodemographic analysis}

In the present case study, the patient was a 45 years old male (Figure 1). The patient belonged to Sirmour, Himachal Pradesh and

${ }^{*}$ Correspondence to: Jaimanti Bakshi, Department of Otolaryngology and Head Neck Surgery (ENT), Post Graduate Institute of Medical Education and Research (PGIMER) Chandigarh, India, Tel: 9855827931; E-mail: drjayabakshi@ymail.com

Key words: paragangliomas, vagal, carotid; tumor, surgery, radiotherapy

Received: February 01, 2019; Accepted: February 19, 2019; Published: February 22, 2019 
was watchman by profession. The patient came with the complaint of swelling in the upper left region of the neck. The patient revealed that swelling is present from last three years and, the swelling was insidious at onset and progressed gradually.

\section{Clinical examination}

Patient was a rare case of multiple paragangliomas having a single $6 \times 5 \mathrm{~cm}$, well defined ovoid, firm swelling in the left upper part of the neck. The swelling is located at mastoid tip superiorly, at the level of hyoid inferiorly, at an angle of mandible anteriorly and $5 \mathrm{~cm}$ from angle of the mandible. The swelling was non-tender, non-pulsatile and mobile only in horizontal direction. Overlying skin was healthy, and no complaint of pain and other symptoms was reported. On physical examination, no other such swelling was observed in the patient body. No family history of similar disease was reported.

\section{Radiological assessment}

Contrast enhanced computed tomography (CECT) of neck revealed enhancing lesson at the level of bilateral carotid bifurcations causing splaying of bilateral carotid bifurcations. DSA shous tumor blush at the level of bilateral carotid bifurcations more on the left than right side showing characteristic Lyris sign described in literature (Figures 2 and 3).

\section{Surgical findings}

In a pre-operative diagnosis, the swelling was identified as a carotid body tumor. Intra-operative findings revealed that it involved both the vagal and carotid body. Both the tumors were present on the left side and were excised during the surgery successfully (Figure 4). Post-operatively the swelling was described as bilateral carotid paragangliomas with left vagal paragangliomas. The patient remained admitted after surgery and was discharged after removal of sutures on 7 th day.

\section{Follow up analysis}

Follow up of the patient revealed healthy wound and indirect laryngoscopy showed left vocal cord palsy with no aspiration. Speech therapy then was prescribed, and the patient was followed up for two years for the possibility of recurrence.

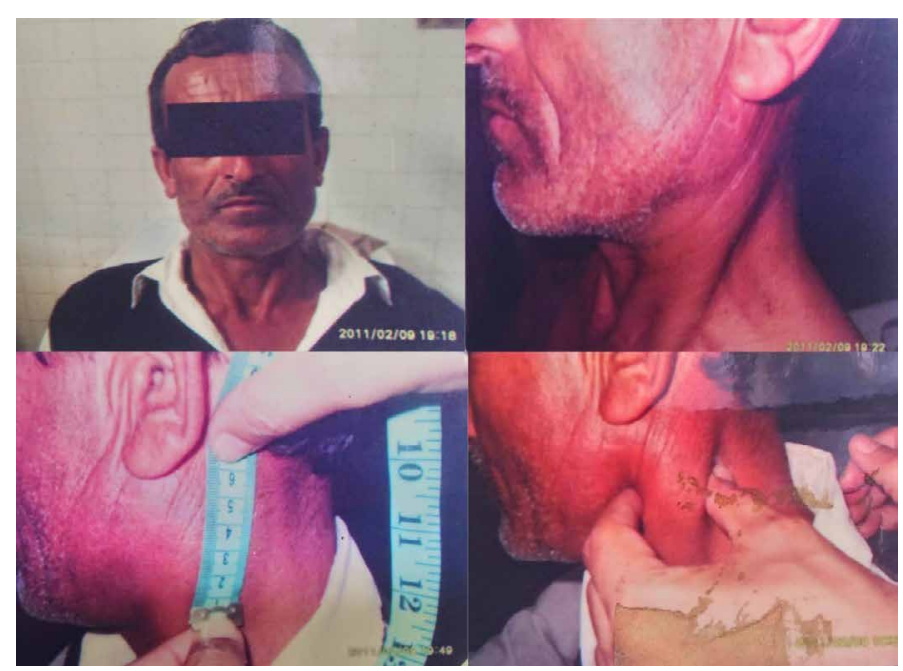

Figure 1. Clinical examination showing swelling on the upper left region of neck

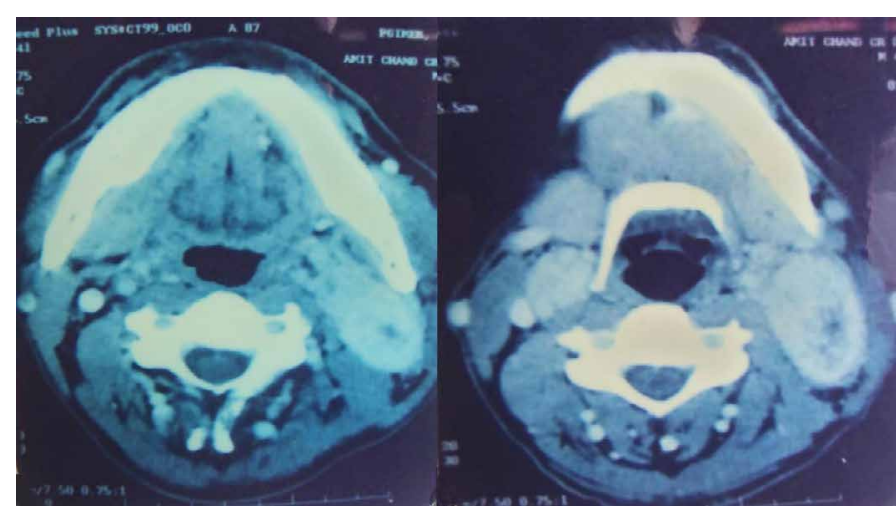

Figure 2. CECT scan showing the enhancing mass lesions in the left and right carotid bifurcations

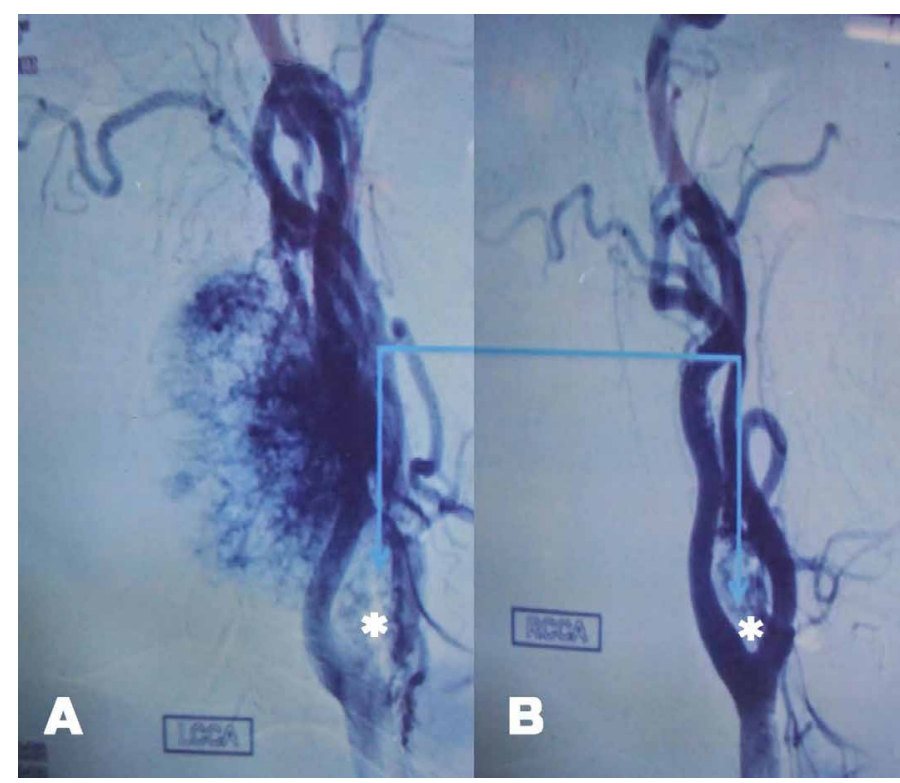

Figure 3. Left common carotid bifurcate (A) and Right common carotid bifurcate (B) showing the Lyre's sign $\left(^{*}\right)$

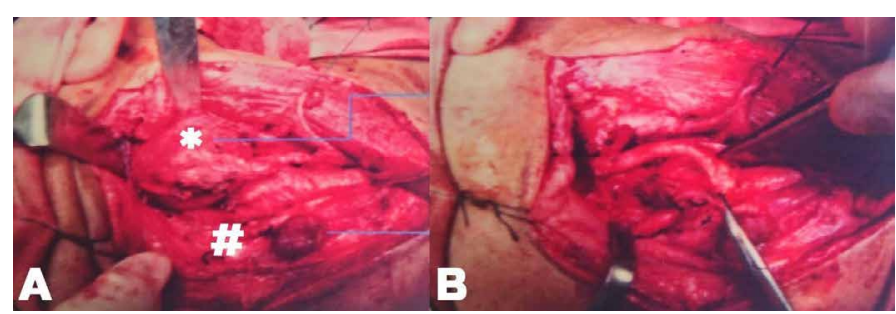

Figure 4. Intra-operation (A) picture showing vagal paragangliomas $(*)$ and carotid paragangliomas (\#) which was remove successfully post-operatively as showed in picture (B)

\section{Discussion}

The existence of multiple paragangliomas involving carotid and vagal region are extremely rare [6], and to the best of our knowledge, the present study represents the second case of the same in the world. An earlier study has reported that the incidence of paragangliomas is more in individual living at high altitudes and who are affected by chronic obstructive pulmonary disease [6]. The patient in the present study was also living in high altitude. Earlier, the paragangliomas were reported to occur five times more frequently in female compared 
to the males [6] and more frequently in middle age patients [7] to which contradictory with present case report in which a 65 -year-old male was reported with paragangliomas. The family history of the disease is considered as a potential risk factor in case of paragangliomas, and around $50 \%$ of cases in earlier studies were reported to have a family history of the disease [6]. However, in the present study, the patient did not have any family history of the disease. The most frequent symptoms associated with the paragangliomas in the neck region reported were palpable neck mass $(55 \%)$, tinnitus (18\%), and cranial nerve palsies (16\%) [9]. In the present study, we also reported the neck mass, but tinnitus and palsies were absent. Patient in the present study reported having no complaints of pain which is consistent with the previous studies $[6,10]$.

Accurate diagnosis of paraganglioma is thought to be difficult, and therefore both clinical findings and radiographic studies are recommended in such cases [7]. Noninvasive techniques such as computerized tomography (CT), magnetic resonance imaging (MRI) or positron emission tomography (PET) can be combined with clinical examination for accurate characterization of the tumor [9]. In the present study also, we used the contrast-enhanced CT scan to see the extent of paragangliomas in the neck region. In some cases, the conventional angiography or digital subtraction angiography was also done to demonstrate the vascular supply of paraganglioma [6] which was also done for the present case.

Although the surgical resection is believed to be the definitive treatment for paragangliomas, difficulties may arise in cases where tumor are inoperable [9]. Also, the surgery generally requires sacrificing the vagus nerve and is associated with other nervous lesions. Therefore, vagal, hypoglossal and glossopharyngeal paralysis are common postoperative complications [7]. In the present case, no such complication was reported postoperatively. Even after successful surgery, there is always the chance of recurrence [9] which was also not seen in the present case in the follow up of two years. Alternative to surgery, radiation therapy is another choice of treatment for arresting the growth of tumor in cases where the patients were inoperable [7] or at high risk for surgery due to tumor size, patient age, co-morbidity conditions, preoperative cranial nerve involvement, anticipated postoperative cranial nerve paralysis, patency of the circle of Willis to provide for cross-cerebral circulation, multiplicity of paragangliomas, and the involvement of local organs (esophagus, soft palate, and larynx) and symptoms $[11,12]$. Also, it has been reported that the radiation can lead to neurologic sequelae and rarely can destroy the tumor [7] because tumors arising from the vagus and carotid body were thought to be radioresistant [11]. However, monolateral radiotherapy can be indicated after contralateral surgery in order to avoid bilateral vagus nerve damage [7]. Also, the gamma knife radiosurgery and CyberKnife stereotactic radiotherapy are thought to provide a higher degree of accuracy and precision than conventional radiotherapy [6].

\section{Conclusion}

The present study represents the sporadic case of paragangliomas involving both the carotid body and vagal region. The clinical examination and radiological studies were done before surgery. The patient was treated successfully with the surgery and recurrence was not reported in two years to follow up. Our study will help the clinicians in designing the treatment strategies for such complicated cases of multiple paragangliomas.

\section{Compliance with ethical standards}

Written informed consent was obtained from the patient mentioning the permission to use clinical data and photographs for research publication purpose.

\section{Source of funding}

PGIMER has provided the facilities for performing surgery and other diagnostic facilities. The work in this research is also supported by the Junior Research Fellowship (JRF) provided by ICMR, New Delhi wide file no. 3/13/JRF -2015/HRD.

\section{References}

1. Xiao Z, She D, Cao D (2015) Multiple paragangliomas of head and neck associated with hepatic paraganglioma: a case report. BMC Med Imaging 15: 38. [Crossref]

2. Seo MC, Lee KS, Kim CJ, Kim SY (2000) Multiple Paragangliomas: Three Cases. Korean Otolaryngol Head Neck Surg 43: 442-446.

3. Ertz-Archambault NM, Van Gompel JJ, Neff BA, Kasperbauer JL, Shamoun FE (2016) What happens in vagus: a case of recurrent paraganglioma with malignan transformation and an updated treatment algorithmâ€. J Surg Case Rep 2016. [Crossref]

4. Davidovic LB, Djukic VB, Vasic DM, Sindjelic RP, Duvnjak SN (2005) Diagnosis and treatment of carotid body paraganglioma: 21 years of experience at a clinical center of Serbia. World J Surg Oncol 3: 10. [Crossref]

5. Wieneke JA, Smith A (2009) Paraganglioma: carotid body tumor. Head Neck Pathol 3: 303-306. [Crossref]

6. Del Guercio L, Narese D, Ferrara D, Butrico L, Padricelli A, et al. (2013) Carotid and vagal body paragangliomas. Transl Med UniSa 6: 11-15. [Crossref]

7. Caldarelli C, Iacconi C, Della Giovampaola C, Iacconi P, Beatrice F (2007) Vaga paragangliomas: two case reports. Acta Otorhinolaryngol Ital 27: 139-143. [Crossref]

8. Bakshi J, Mohammed AW, Lele S, Nada R (2016) Ganglioneuromas involving the hypoglossal nerve and the vagus nerve in a child: Surgical difficulties. Ear Nose Throat J 95: E22-E24. [Crossref]

9. Tonyukuk V, Emral R, Temizkan S, Sertçelik A, Erden I, et al. (2003) Case report patient with multiple paragangliomas treated with long acting somatostatin analogue. Endocr J 50: 507-513. [Crossref]

10. Langerman A, Athavale SM, Rangarajan SV, Sinard RJ, Netterville JL (2012) Natura history of cervical paragangliomas: outcomes of observation of 43 patients. Arch Otolaryngol Head Neck Surg 138: 341-345. [Crossref]

11. Pandey M, Chandramohan K, Sebastian P, Ramachandran K (2002) An unusua bilateral cervical paraganglioma: a case report. Int J Oral Maxillofac Surg 31: 334-346. [Crossref]

12. Myssiorek D, Persky M (2016) Treatment of carotid paraganglioma. Oper Tech Otolayngol Head Neck Surg 27: 30-35.

Copyright: (C2019 Bakshi J. This is an open-access article distributed under the terms of the Creative Commons Attribution License, which permits unrestricted use, distribution, and reproduction in any medium, provided the original author and source are credited. 